\title{
Hitting the Dartboard from 40,000 Feet: A Better Chance with Your Eyes Open!
}

\author{
Bruce R. King, FRACP, Ph.D.,, ${ }^{1,2}$ Peter W. Goss, FRACP, ${ }^{3}$ Megan A. Paterson, C.D.E., \\ Patricia A. Crock, FRACP, ${ }^{1,2}$ and Donald G. Anderson, FRACP ${ }^{1,2}$
}

\section{Dear Editor:}

$\mathbf{T}$ HANK YOU FOR THE OPPORTUNITY to address the questions raised in the editorial "Hitting the dartboard from 40,000 feet" ${ }^{\prime 1}$ commenting on our article "Changes in altitude cause unintended insulin delivery from insulin pumps: mechanisms and implications." ${ }^{2}$ We agree with Prof. Hirsch's insightful view into the vagaries of glycemic control. We hope that understanding how altitude affects the physical properties of insulin will remove a factor that impairs glycemic control.

The study was performed in response to a clinical problem of two children who were regular air travelers with tight glycemic control who consistently developed hypoglycemia 1-2 h after takeoff. We are now aware of over 50 insulin pump users (children and adults) who attributed their regular flightassociated hypoglycemia to the vagaries of diabetes and never raised the issue with their clinicians.

This study demonstrated that insulin conforms to the laws of physics described by William Henry in $1803^{3}$ and Robert Boyle in 1662. ${ }^{4}$ These laws accurately and repeatedly predicted how much additional insulin was delivered from the insulin pumps when the pressure decreased (ascent in a plane) and the deficit that occurred when the pressure was increased (descent). ${ }^{2}$ We demonstrated that the cause of this unintended insulin delivery was due to air coming in and out of solution (Henry's law $\left.{ }^{3}\right)$. The research was appropriately powered and statistically significant.

We agree with Prof. Hirsch that the described phenomenon needs to be kept in perspective as the unintended insulin delivery is relatively small. Hence the resultant hypoglycemia in tightly controlled adult patients will be mild but will be potentially more significant for children.

Nonetheless, unintended insulin delivery with pressure changes is predictable and preventable, and awareness of the phenomenon empowers the patient to intervene. The recommendations were developed following lengthy consideration of the study findings and consultation with airlines, pilots, and aviation physicians. ${ }^{2}$

The reasoning for the recommendations is as follows:

1. The pump should contain $<1.5 \mathrm{~mL}$ of insulin. The excess insulin delivered is proportional to the amount of insulin in the cartridge.
2. Disconnect the pump before takeoff. The excess insulin is delivered as pressure drops during ascent.

3. When the plane is at cruising altitude $(30 \mathrm{~min}$ after takeoff in all commercial flights), check the cartridge of bubbles and then reconnect the pump. This removes the bubbles so the pump will function normally during descent (because there are no bubbles to be reabsorbed).

4. Once the plane has landed, disconnect the pump and bolus through 2 units and then reconnect the pump. This ensures there is no deficit and that the pump will function normally.

5. In the case of an in-flight decompression emergency disconnect the pump. During large decompressions the pump will deliver a large dose of insulin.

Prof. Hirsch highlighted that in decompression emergencies a person may be distracted by the oxygen masks dropping down and the plane going into a rapid and anxiety-provoking dive to attain a cabin pressure equivalent to 8,000 feet. However, an emergency plan, as we have recommended, will empower the patient to respond in a logical way-even if he or she failed to disconnect during the emergency, the individual would be aware that additional insulin was inadvertently delivered.

Prof. Hirsch was concerned that disconnecting the insulin pump risks ketoacidosis. We recommend disconnecting for only $30 \mathrm{~min}$. Insulin pump users disconnect the pump frequently on a daily basis without resultant ketoacidosis (i.e., showering, exercise), and there is no evidence that air travel should increase the risk of forgetting to reconnect. Conversely, if a person ignored the recommendations, it could lead to ketosis. If there was a 1.4 unit deficit and the basal rate was 0.5 units $/ h$, then there is potentially $3 \mathrm{~h}$ of non-delivery of insulin. We are aware of a 42-year-old woman who reported hypoglycemia followed by ketosis post-flight. She found the infusion line to be full of air.

Prof. Hirsch suggested that disconnecting could impair glycemic control.

A 30-min pump disconnection would lead to an insulin deficit of 0.5 units, assuming a basal rate of 1 unit/h. If we assume a sensitivity factor of $72 \mathrm{mg} / \mathrm{dL}$ per unit of insulin, then this would cause a $36 \mathrm{mg} / \mathrm{dL}$ increase in blood glucose

\footnotetext{
${ }^{1}$ John Hunter Children's Hospital, University of Newcastle, Newcastle, New South Wales, Australia.

${ }^{2}$ Hunter Medical Research Institute, Newcastle, New South Wales, Australia.

${ }^{3}$ Gippsland Paediatrics Diabetes Unit, Sale, Victoria, Australia.
} 
level. Our study did not comment on compensating for this blood glucose level increase because it is a clinical decision that requires interpretation of the factors described by Prof. Hirsch.

Conversely, we contend that failure to follow the recommendations could impair glycemic control. Hypoglycemia is frequently overtreated, producing hyperglycemia, and if it was followed by a 1.4 unit insulin deficit, then the potential for hyperglycemia is significant.

Our recommendations to manage insulin pumps during air travel have been locally active for over 12 months with no reported problems. By following our recommendations, the predictable hypoglycemia during air travel in the index cases has resolved.

Thus, we demonstrated that during air travel there was reproducible and quantifiable excess insulin delivery during ascent and deficient insulin delivery during descent from insulin pumps. This information allows the clinician and pump user to be informed. If we are trying to hit a dartboard of glycemic control, then it is much easier with our eyes open.

\section{References}

1. Hirsch IR: Hitting the dartboard from 40,000 feet. Diabetes Technol Ther 2011;13:981-982.

2. King BR, Goss PW, Paterson MA, Crock PA, Anderson DG: Changes in altitude cause unintended insulin delivery from insulin pumps: mechanisms and implications. Diabetes Care 2011;34:1932-1933.

3. Henry's Law. en.wikipedia.org/wiki/Henry\%27s_law (accessed December 2, 2010).

4. Boyle's Law. en.wikipedia.org/wiki/Boyle\%27s_law (accessed December 2, 2010).

Address correspondence to: -Bruce R. King, FRACP, Ph.D. John Hunter Children's Hospital Locked Bag No: 1

Hunter Regional Mail Centre Newcastle, NSW, Australia 2310

E-mail: Bruce.King@hnehealth.nsw.gov.au 